\title{
Methylphenidate Administration to Adolescent Rats Determines Plastic Changes on Reward-Related Behavior and Striatal Gene Expression
}

\author{
Walter Adriani ${ }^{1,5}$, Damiana Leo ${ }^{2,5}$, Dario Greco ${ }^{3}$, Monica Rea', Umberto di Porzio², Giovanni Laviola' \\ and Carla Perrone-Capano*,2,4 \\ 'Department of Cell Biology \& Neurosciences, Behavioral Neuroscience Section, Istituto Superiore di Sanità, Roma, Italy; ${ }^{2}$ Institute of Genetics \\ and Biophysics 'A. Buzzati Traverso', CNR, Napoli, Italy; ${ }^{3}$ Institute of Biotechnology, University of Helsinki, Helsinki, Finland; ${ }^{4}$ Department of \\ Pharmacobiology, University of Catanzaro 'Magna Graecia', Roccelletta di Borgia (CZ), Italy
}

\begin{abstract}
Administration of methylphenidate (MPH, Ritalin $\left.{ }^{\mathbb{R}}\right)$ to children with attention deficit hyperactivity disorder (ADHD) is an elective therapy, but raises concerns for public health, due to possible persistent neurobehavioral alterations. Wistar adolescent rats (30 to 46 day old) were administered MPH or saline (SAL) for 16 days, and tested for reward-related and motivational-choice behaviors. When tested in adulthood in a drug-free state, MPH-pretreated animals showed increased choice flexibility and economical efficiency, as well as a dissociation between dampened place conditioning and more marked locomotor sensitization induced by cocaine, compared to SALpretreated controls. The striatal complex, a core component of the natural reward system, was collected both at the end of the MPH treatment and in adulthood. Genome-wide expression profiling, followed by RT-PCR validation on independent samples, showed that three members of the postsynaptic-density family and five neurotransmitter receptors were upregulated in the adolescent striatum after subchronic MPH administration. Interestingly, only genes for the kainate 2 subunit of ionotropic glutamate receptor (Grik2, also known as KA2) and the 5-hydroxytryptamine (serotonin) receptor 7 ( $\mathrm{Htr} 7$ ) (but not $G A B A_{A}$ subunits and adrenergic receptor $\left.\alpha / b\right)$ were still upregulated in adulthood. CAMP responsive element-binding protein and Homer la transcripts were modulated only as a long-term effect. In summary, our data indicate short-term changes in neural plasticity, suggested by modulation of expression of key genes, and functional changes in striatal circuits. These modifications might in turn trigger enduring changes responsible for the adult neurobehavioral profile, that is, altered processing of incentive values and a modified flexibility/habit balance.

Neuropsychopharmacology (2006) 3 I, 1946-1956. doi: I0.1038/sj.npp. I300962; published online 23 November 2005
\end{abstract}

Keywords: ADHD; dopamine; flexibility; gene expression profiling; habit; PSD family

\section{INTRODUCTION}

Methylphenidate (MPH) is a psychostimulant currently prescribed to children diagnosed with attention deficit hyperactivity disorder (ADHD), one of the most common chronic neurobehavioral diseases of childhood (Swanson et al, 1998). ADHD is viewed as an executive dysfunction, according to the dominant model. However, alternative accounts present ADHD as a motivational dysfunction, characterized by attempts to escape or avoid delay, arising

\footnotetext{
*Correspondence: Professor C Perrone-Capano, Institute of Genetics and Biophysics 'A. Buzzati Traverso', CNR, Via Pietro Castellino I I I, Naples 80 I3I, Italy, Tel: + 3908 |6132362, Fax: + 3908|6132350,

E-mail: perrone@igb.cnr.it

${ }^{5}$ Both authors have equally contributed to this work.

Received 7 July 2005; revised 3 October 2005; accepted 4 October 2005

Online publication: 12 October 2005 at http://www.acnp.org/citations/ Npp 10 I205050440/default.pdf
}

from altered reward processes in fronto-striatal circuits (Sagvolden and Sergeant, 1998; Sonuga-Barke, 2003). MPH interacts with the same brain pathways activated by drugs of abuse, producing striatal dopamine (DA) overflow similar to cocaine (Swanson and Volkow, 2002). Hence, exposure to MPH during adolescence is a major concern for public health, due to possible long-term effects (Carlezon and Konradi, 2004).

Studies in rodents are useful in defining molecular and behavioral responses to MPH. Recent reports in rats showed that exposure to MPH during adolescence causes behavioral changes enduring into adulthood, including enhanced psychomotor responses to cocaine and increased cocaine self-administration (Brandon et al, 2001), reduced rewarding power and increased sensitivity to the aversive effects of cocaine (Andersen et al, 2002), depressive-like effects in the forced-swim test, and attenuated locomotor habituation (Carlezon et al, 2003). Furthermore, adult animals treated with MPH during adolescence are less responsive to natural 
rewards, showing increased stressful and anxiety-like behaviors (Bolanos et al, 2003).

We further characterized the enduring effects of adolescent MPH exposure on incentive properties of cocaine and on development of locomotor sensitization to this drug. We also extended behavioral end points to the study of motivation-driven choice. Decision-making behavior impinges on cortical/striatal pathways, accounting for processing of incentive values when choosing between two alternatives (Montague and Berns, 2002; Glimcher and Rustichini, 2004; Schultz, 2004). We gave animals a choice between a smaller certain reinforcer and a larger but probabilistic one, a protocol also used to measure impulsivity (Mobini et al, 2000; Evenden, 1999). Economical considerations play, however, a major role in such a protocol. Indeed, rats classically shift from a larger but probabilistic reinforcer towards a smaller but certain one when foraging opportunities are not a limiting factor. Conversely, it has been shown that 'risky' choices do actually increase if the total number of foraging possibilities is reduced under the scheduled test conditions (Hastjarjo et al, 1990; Kaminski and Ator, 2001). The contingencies used in the present study actually induced an economically driven shift towards the 'risky' choice as a reaction to largereward rarefaction. We assessed the ongoing and enduring effects of MPH treatment during adolescence in this task. We expected to detect MPH-induced changes in economical evaluation and comparison of the two actual outcomes, associated with either choice.

Molecular evidence suggests that repeated psychostimulant exposure causes stable changes in gene expression and persistent alterations in dendritic morphology within the mesocorticolimbic DA system, the main pathway of the brain reward circuitry (Nestler, 2004; Robinson and Kolb, 2004). Similar plastic changes in reward-related circuits might also occur after repeated $\mathrm{MPH}$, especially with adolescent exposure (Carlezon and Konradi, 2004). Recent reports showed increased dynorphin expression in the dorsal striatum at the end of the adolescent treatment (Brandon and Steiner, 2003) and increased cAMP responsive element-binding protein $(C R E B)$ expression within the nucleus accumbens (NAc) shell in adulthood (Andersen et al, 2002). Acute exposure to MPH is known to alter the expression of $\mathrm{c}$-fos and substance $P$ preferentially in the dorsal striatum (Yano and Steiner, 2004). However, the whole molecular consequences of chronic exposure to $\mathrm{MPH}$ during critical periods of development are poorly understood (Carlezon and Konradi, 2004). Our work was aimed at combining behavioral and molecular approaches, to identify the short- and long-term effects of subchronic treatment with MPH. To study gene-expression changes, we have used microarray technology, to screen the expression of more than 15000 transcripts in the rat striatum simultaneously, and then RT-PCR, to validate variation of expression for selected genes.

\section{MATERIALS AND METHODS}

Experimental protocols were approved by institutional authorities and are in close agreement with European Community Directives and with the Italian Law. All efforts were made to minimize animal suffering, to reduce the number of animals used, and to use alternatives to in vivo testing.

\section{Drugs}

MPH (CIBA-GEIGY, Italy) was dissolved in saline (SAL, $\mathrm{NaCl} 0.9 \%$ ) and injected i.p. in a volume of $1 \mathrm{ml} / 200 \mathrm{~g}$ body weight. Cocaine (COC) was dissolved in SAL and injected s.c. in a volume of $1 \mathrm{ml} / 100 \mathrm{~g}$ body weight. The COC dose was chosen based on previous studies (Laviola et al, 1995). Rats belonging to control groups were injected with SAL.

\section{Cocaine Conditioning and Sensitization after Adolescent MPH Exposure}

Wistar male rats (Harlan, Italy) were tested in adulthood in a classical place-conditioning test, with a three-chamber apparatus and a 'biased' procedure. Animals received three cocaine ( 0 or $10 \mathrm{mg} / \mathrm{kg}$ s.c.) injections in the paired chamber and three saline injections in the unpaired chamber. Animals were then tested for place preference in a drugfree state (see Supplementary information).

\section{Choice Behavior Following Adolescent MPH Exposure}

Subjects and rearing conditions. Wistar pregnant female rats (Harlan, Italy) were housed in an air-conditioned room (temperature $21 \pm 1{ }^{\circ} \mathrm{C}$, relative humidity $60 \pm 10 \%$ ), with a 12-h light-dark cycle (lights on at $8.00 \mathrm{am}$ ). Water and food (Enriched Standard Diet, Mucedola, Settimo Milanese, Italy) were available ad libitum. The day of delivery was considered as postnatal day (pnd) zero, pups being culled to six males and two females. Pups were then weaned on pnd 21 and housed in groups of two nonsiblings, according to sex. Only two male subjects per litter were used in this experiment, the other four male subjects being used for gene-expression analyses (see below). Within each litter, one sibling was assigned to the SAL control group and the other to the MPH treatment group, according to a split-litter design (Zorrilla, 1997). The MPH treatment $(2 \mathrm{mg} / \mathrm{kg}$ i.p. once daily) was administered during adolescence (from pnd 30 to 46). Animals were tested for motivational-choice behavior during MPH or SAL treatment (pnd 30-46), and again in adulthood (pnd $>60$ ) in a drug-free state.

Two-choice operant-behavior test. Animals were tested in a probability-based reward-rarefaction protocol (see Introduction). Before the schedule started, animals were foodrestricted for 2 days, to keep them at $80-85 \%$ of their free-feeding weight in order to increase their motivation to work for food delivery. Each animal was then placed daily in a computer-controlled operant chamber (Coulbourn Instruments, USA), provided with two nose-poking holes, a chamber light, a feeder device, a magazine where pellets (45 mg, BioServ, USA) were dropped, and a magazine light. The nose-poking in either hole was detected by a photocell and was recorded by a computer, which also controlled food delivery. After the 20-min session, animals were returned to their home cage, where they were given standard chow (approximately $10 \mathrm{~g} / \mathrm{each}$ ).

During the training phase ( 1 week), nose-poking in one of the two holes (called 'SMALL \& CERTAIN' hole, H1) resulted in 
the delivery of one pellet of food, whereas nose-poking in the other hole ('LARGE \& UNCERTAIN' hole, H5) resulted in the delivery of five pellets of food. After nose-poking and before food delivery, the chamber light was turned on for $1 \mathrm{~s}$. Following the food delivery, the magazine light was turned on for $25 \mathrm{~s}$, during which nose-poking was recorded, but was without any scheduled consequence (time-out).

During the testing phase (1 week), a probabilistic dimension was associated to the delivery of the five pellets following $\mathrm{H} 5$ nose-poking. The chamber and the magazine lights were turned on following the previous schedule. However, sometimes the delivery of food could be omitted according to a given level of probability (' $p$ ' = percentage of actual food delivery) controlled by the computer. The probability level was kept fixed for each daily session, and was decreased progressively over subsequent days. A first dependent variable was the choice (\%) for the certain reinforcer, namely percentage of $\mathrm{H} 1$ over total $\mathrm{H} 5+\mathrm{H} 1$ choices. A second dependent variable was the slope of the preference-probability curve, calculated with the Microsoft Excel 'slope' function, using $\mathrm{H} 1$-preference as the $Y$-axis data and (100-' $p$ ') as the $X$-axis data.

Probability values to be imposed can be divided into two distinct fields, separated by the indifference-point ' $p$ ' value (calculated as 'small reward size'/'big reward size', ie $20 \%$ in our work). In the range of ' $p$ ' values before the indifference point $(100 \%>p>20 \%)$, the risk of losing large reinforcement is mild relative to its size. Under these conditions, it is still 'economically' convenient for rats to choose H5 (the average outcome being still between 5 and 2.5 pellets per nose-poking) over $\mathrm{H} 1$ hole (only one pellet per nosepoking). Hence, animals may be expected to shift towards increased $\mathrm{H} 5$ demanding, as a reaction against a mild uncertainty challenge. In the range of ' $p$ ' values beyond the indifference point $(20 \%>p>0 \%)$, it becomes 'economically' convenient for rats to choose $\mathrm{H} 1$ (one certain pellet per nose-poking) over the $\mathrm{H} 5$ hole (the average outcome being less than one pellet per nose-poking). Under these conditions, animals classically shift from the probabilistic towards the certain reward.

On this basis, we imposed two ranges of ' $p$ ' values: either above or below the indifference point. Animals were tested the first time during adolescence, to assess the subchronic effects of actual MPH treatment on levels of motivationalchoice behavior. Operant-behavior sessions started at least $2 \mathrm{~h}$ after MPH or SAL injection (Adriani et al, 2004). During this 2 -week testing period, ' $p$ ' decreased regularly from 100 to $50 \%$. This range was chosen as a mild rarefaction challenge. The same subjects were tested again in a drugfree state in adulthood (ie 4 weeks after the end of treatment). This was aimed to assess the long-term carryover consequences of adolescent MPH exposure on levels of adult self-control behavior. During this 1-week testing period, rats were kept for a few days at $p=100 \%$, to reobtain a stable baseline. Then, ' $p$ ' decreased regularly from 20 to $12.5 \%$. This range was chosen as a stronger rarefaction challenge.

Design and data analysis. Data were analyzed by randomized-block ANOVA. The general design of the experiment was two treatments $(\mathrm{MPH} v \mathrm{SAL}) \times$ session (various levels of probability ' $p$ ' fixed for each session). All variables were within-litter factors. Multiple comparisons within significant interactions were performed with the Tukey HSD test.

\section{Expression Profiling of Striatal Genes Following Adolescent MPH Exposure}

Subjects and rearing conditions. The subjects used for this experiment were four male rats per litter, siblings of those used in the previous experiment. Nine litters were used ('first' batch): within each litter, two siblings were assigned to a SAL control group and two others to an MPH treatment group, according to a split-litter design. The MPH treatment ( 0 or $2 \mathrm{mg} / \mathrm{kg}$ i.p. once daily) was administered during adolescence (from pnd 30 to 46). Two sibling animals were killed two hours following the last SAL or MPH injection during adolescence (ie at pnd 46), and the other two siblings were killed during adulthood (at pnd $>90$, ie at least 8 weeks after the last drug injection). Only adolescent sibling rats were used for the microarray experiments. In a separate ('second') batch of animals, five pairs of adolescent siblings were repeatedly treated as described above ( 0 or $2 \mathrm{mg} / \mathrm{kg}$ i.p. from pnd 30 to 46), to obtain an RT-PCR validation of microarray data on independently treated animals. Finally, four pairs of adolescent siblings were injected only at pnd 46 with SAL or MPH (0 or $2 \mathrm{mg} / \mathrm{kg}$ i.p.) and used to check the effects of a single acute injection of the drug. All animals were killed two hours after injection. The brain was quickly removed and rinsed in phosphate-buffered saline. The striatal complex, including both dorsal and ventral portions, was carefully dissected from the whole brain and processed for RNA isolation.

RNA isolation. Independent RNA samples from the bilateral striatal complex of nonsibling rats were separately extracted for each experimental group (adolescent and adult SAL- and MPH-treated animals) using the TriReagent (Sigma-Aldrich, Milan, Italy), according to the manufacturer's instructions. After RNA extraction, any remaining genomic DNA was digested for 30 min using the DNA Free system (Ambion Inc., Milan, Italy), according to the manufacturer's instructions. An additional clean-up of total RNA was performed using the RNeasy kit (Qiagen, Milan, Italy). RNA concentration was spectrophotometrically determined, and RNA integrity was confirmed by agarose gel electrophoresis. RNA samples were further processed for microarray hybridization (from adolescent rats) or for RTPCR experiments (from adolescent and adult rats).

Probe preparation and microarray hybridization. To minimize the biological variability, RNAs from nine adolescent animals per experimental group were used ('first' batch). Samples were pooled after purification, leading to three independent pooled-RNA samples (each deriving from three different animals) for both the SAL- and the MPH-treated group. cDNA synthesis, labeling, and hybridization were performed on the three pooled-RNA samples. Using the protocol supplied by the manufacturer (Affymetrix, Santa Clara, CA), double-stranded cDNA was synthesized from total RNA and was used to obtain biotinlabeled cRNA by an in vitro transcription reaction (ENZO Diagnostics, Farmingdale, NY). Biotin-labeled cRNA was 
fragmented and hybridized with Affymetrix RAE230A rat genome GeneChip microarrays ( $n=3$ per group), according to the manufacturer's protocol, after verifying the quality of the biotin-labeled cRNA on a Test Chip (Affymetrix).

Microarray data analyses. Scanning of the slides was accomplished according to the manufacturer's instructions (see Supplementary information for further details).

Reverse transcription and semiquantitative PCR. RT-PCR analyses were performed on RNA samples from individual rats of both SAL- and MPH-treated groups. These analyses were run on the 'first' batch of animals $(n=9$ for rats killed during adolescence and also used for microarrays; $n=9$ for rats killed during adulthood) and on the 'second' independent batch of SAL- and MPH-injected animals, killed during adolescence ( $n=5$ for rats treated subchronically; $n=4$ for rats treated acutely). See Table 1 and detailed methods in Supplementary information.

\section{RESULTS}

\section{Cocaine Conditioning and Sensitization after Adolescent MPH Exposure}

Cocaine-induced sensitization of locomotor activity in adulthood. During the pairing period (days 1, 2, 3 of the schedule; see Figure 1), adult animals receiving COC expressed significantly higher activity levels than animals receiving SAL in the paired compartment (drug, $\mathrm{F}(1,30)=49.6, p<0.001)$. The two pairing groups were analyzed separately. Within the SAL-pairing group, MPH pretreatment produced no carryover effects on basal levels of locomotion (no significant pretreatment effects, $\mathrm{F}(1,14)=1.40, \mathrm{NS})$. Conversely, within the COC-pairing group, MPH-pretreated rats showed significantly higher hyperactivity levels (pretreatment, $\mathrm{F}(1,16)=14.6, p<0.01$ ), when compared to SAL-pretreated controls. Specifically, MPH pre-exposure in adolescence markedly potentiated the response to COC in adulthood. Furthermore, on examining the day-by-day values, the activity levels of the SAL-pretreated group did not vary across days (day, $\mathrm{F}(2,10)=0.919$, NS), suggesting very low levels of sensitization. Conversely, a steady increase in COC-induced hyperactivity was evident over days within the MPHpretreated group (day, $\mathrm{F}(2,22)=3.78, p<0.05)$. These results indicate a more marked profile of sensitization to $\mathrm{COC}$ effects in the adult as a consequence of MPH preexposure during adolescence.

Cocaine-induced place-conditioning in adulthood. During the test in a drug-free state (day 4 of the schedule; see Figure 1), animals pretreated with MPH during adolescence showed a quite different profile when compared to the SAL-pretreated ones (pretreatment $\times$ drug, $\mathrm{F}(1,30)=3.59$, $p<0.05)$. Multiple comparisons confirmed that the two SAL-pairing groups did not differ significantly, thus ruling out the potential carryover effects of MPH pre-exposure in basal preference within the apparatus. The two pretreatment groups were then analyzed separately. As for the SAL-pretreatment group, COC pairing produced a significant conditioned preference for the drug-paired environ-

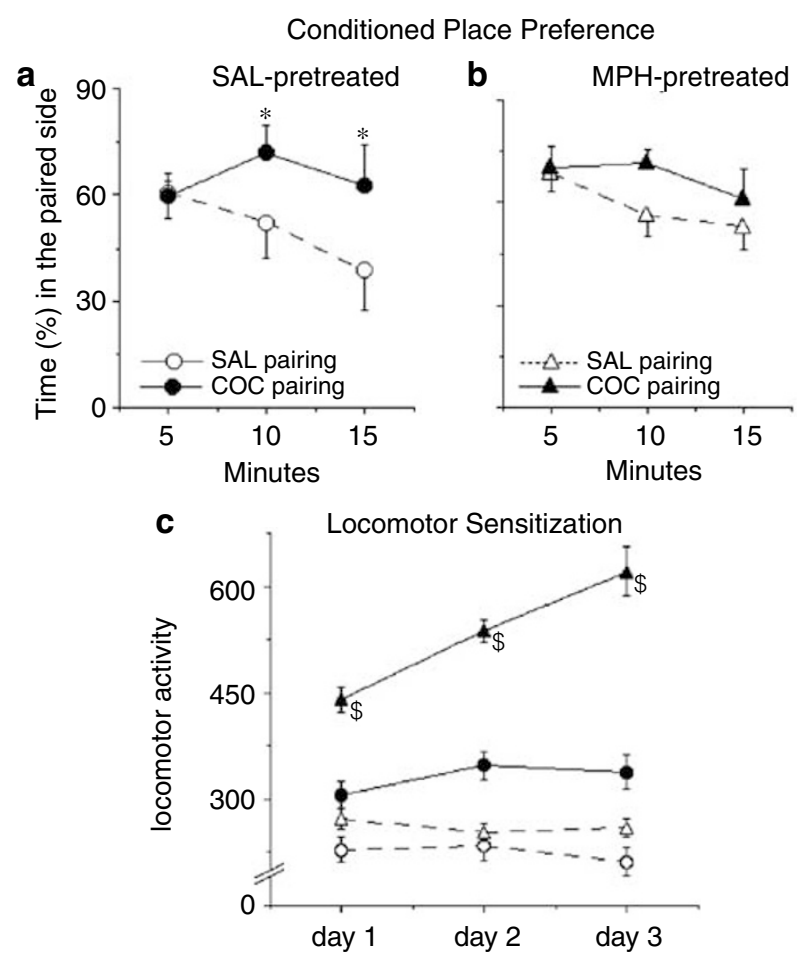

Figure I Cocaine-induced sensitization and place-conditioning tested in adulthood in rats pretreated with SAL (circles) or MPH (triangles) during adolescence (pnd 30-46). On days I, 2, 3 of the schedule (pairing period), half of the animals (COC pairing group) received COC $(10 \mathrm{mg} / \mathrm{kg})$ or SAL before being placed in the paired or unpaired compartments, respectively $(n=|| / \mid 2)$. Conversely, the other half of the animals (SAL pairing group) received SAL before being placed in both compartments $(n=5 / 6)$. On day 4 of the schedule (test), animals were tested for place preference in a drugfree state. Panels a and b: the mean ( \pm SEM) time (\%) spent in the paired compartment on day 4 (place-preference test). $* p<0.05$ when comparing COC with SAL pairing. Panel c: the mean ( \pm SEM) locomotor activity expressed in the paired compartment on days 1, 2, 3 (pairing period). ${ }_{p}<0.05$ when comparing MPH with SAL pretreatment.

ment (drug, $F(1,9)=14.5, p<0.01$ ), when compared to the SAL-pairing control group. Conversely, within the MPH-pretreatment group, no significant conditioned place preference was found between COC- and SAL-pairing (no significant drug effects, $\mathrm{F}(1,21)=1.67$, NS). Specifically, $\mathrm{MPH}$ pre-exposure in adolescence somewhat dampened the ability of COC to induce place conditioning in adulthood.

\section{Choice Behavior Following Adolescent MPH Exposure}

MPH modulation of choice behavior during adolescence. Following 1 week of training with the operant protocol, all rats exhibited a significant preference for the H5 over the H1 hole. However, a certain 'baseline' level (average $18.8 \pm 1.8 \%$ at $p=100 \%$ ) of $\mathrm{H} 1$ nose-poking was always present. This finding indicates that animals were not completely fixed on the more rewarding $\mathrm{H} 5$ choice, but constantly probed the outcome of $\mathrm{H} 1$ choices.

When ' $p$ ' was gradually reduced from 100 to $50 \%$, an interesting profile emerged as a function of ongoing $\mathrm{MPH}$ treatment (see Figure 2). Namely, the SAL-injected controls showed a further decrease in $\mathrm{H} 1$ choice, which reached a level of $11.6 \pm 3.7 \%$ at $p=50 \%$ (half of the previous levels). 
This profile was somewhat expected, based on behavioraleconomy considerations (see Materials and methods).

Conversely, the MPH-injected rats did not show any decrease in $\mathrm{H} 1$ choice, which maintained a constant level $(19.4 \pm 5.9 \%$ at $p=50 \%)$. ANOVA performed on slope data supported this conclusion (main effect of treatment, $\mathrm{F}(1,8)=4.66, p<0.05)$. The preference-probability curve exhibited by SAL-injected controls had a clearly decreasing slope (negative, $-0.248 \pm 0.082$ ). This parameter was conversely rather flat $(0.041 \pm 0.130)$ for MPH-treated siblings.

Carryover effects of adolescent MPH on choice behavior in adulthood. Following a few days of re-exposure to the operant protocol, all rats exhibited a significant preference for the $\mathrm{H} 5$ over the $\mathrm{H} 1$ hole. The 'baseline' level for the $\mathrm{H} 1$ nose-poking was $13.1 \pm 3.8 \%$ at $p=100 \%$. Again, animals exhibited a certain degree of probing for H1-choice outcome. During this testing period, ' $p$ ' was initially reduced to $20 \%$ and then gradually decreased to $12.5 \%$. This schedule provided animals with a stronger challenge, allowing them to express (in)tolerance to large-reward rarefaction (see Figure 2). ANOVA yielded significance for session $(\mathrm{F}(4,32)=5.26, p<0.01)$ and pretreatment $\times$ session interaction $(\mathrm{F}(4,32)=3.25, p<0.05)$. Specifically, the SAL pre-exposure controls showed little and no significant changes in $\mathrm{H} 1$ choice, which reached a level of $17.2 \pm 5.1 \%$ at $p=12.5 \%$. Conversely, the MPH-pretreated rats showed no significant change at $p=20 \%$, followed by a prominent increase in $\mathrm{H} 1$ choice (which reached a level of $32.8 \pm 7.6 \%$ at $p=12.5 \%$ ). This set of findings was confirmed by the ANOVA performed on slope data (main effect of pretreatment, $\mathrm{F}(1,8)=10.5, p<0.05)$. The preference-probability curve exhibited by SAL pre-exposure controls was rather flat $(0.163 \pm 0.345)$, whereas it had a steeply increasing slope (positive, $+2.431 \pm 0.722$ ) for their MPH-pretreated siblings.

Data during adulthood indicate that SAL-pre-exposed subjects showed little or no reaction to a high degree of probabilistic rarefaction. In other words, control animals seem to have learned that, despite a great number of lost rewards, a large food reinforcer will eventually come. These animals preferred to wait for this 'lucky' but 'rare' event, and kept demanding at the $\mathrm{H} 5$ hole. In contrast, MPHpretreated subjects exhibited sensitivity to the enhancement of large-reinforcer rarefaction. Indeed, they reacted by showing a marked shift towards $\mathrm{H} 1$ nose-poking, delivering the smaller but certain reward. Previous MPH administration during adolescence rendered adult animals more sensitive to changes in stochastic loss of reward. In other words, MPH exposure in the adolescent period enhanced the ability to exhibit a reaction against reward rarefaction in adulthood. Since the same animals were tested twice, it is possible that behavior shown in adulthood was somewhat influenced by previous exposure during adolescence. However, it is worth noting that SAL rats, who displayed flexible behavioral adaptation as adolescents, did not change strategy as adults, and conversely for MPH subjects. This observation allows to dismiss the possibility that adult behavior was a mere replication of the adolescent one, and actually strengthens the originality and validity of data obtained at either age.

\section{Expression Profiling of Striatal Genes Following Adolescent MPH Exposure}

Microarray data analyses. To identify the molecular changes elicited by chronic MPH administration, we performed gene profiling of striatal transcripts by a
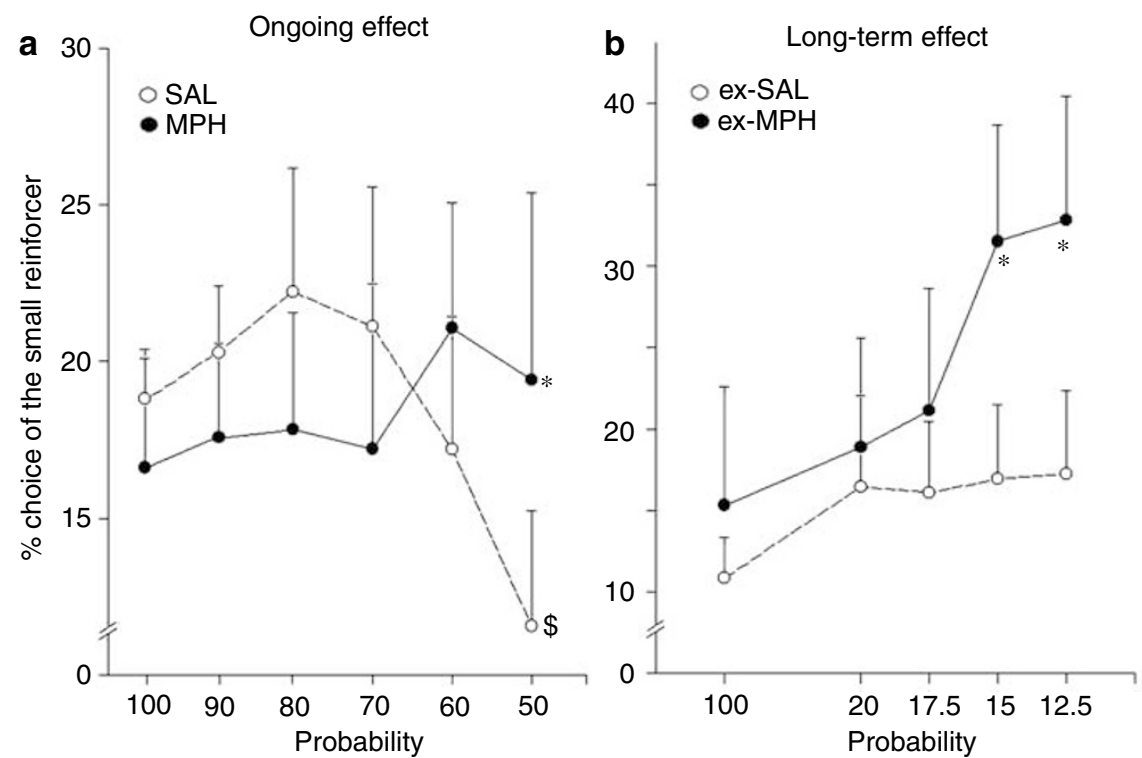

Figure 2 Choice behavior in rats treated with MPH or SAL $(n=9)$ during adolescence (pnd 30-46). Rats were tested twice: during ongoing treatment (a) and in adulthood (pnd $>90, b$ ). Animals had a choice between one certain pellet (by nose-poking at the HI hole) and five uncertain pellets (by nose-poking at $\mathrm{H} 5$ hole), that is, delivered or not with a given probability ' $p$ '. Data represent the mean ( \pm SEM) choice $(\%)$ of the small and certain reinforcer, demanded by nose-poking at $\mathrm{HI}$ hole, as a function of the ' $p$ ' value. The effect of introducing a probabilistic dimension was evident in adolescent controls, while $\mathrm{MPH}$-treated rats were more 'tolerant' to large-reinforcement rarefaction (a). On the contrary, the effect of the probabilistic dimension was evident in adult $\mathrm{MPH}$-pretreated rats, expressing a 'flexible' response, but not in controls, expressing a 'habitual' response (b). ${ }^{\$} p<0.05$ when comparing across sessions with different ' $p$ ' levels; * $p<0.05$ when comparing MPH with SAL treatment. 
genome-wide microarray technique using Affymetrix RAE230A rat genome GeneChips that contain over 15000 probesets, analyzing about 15000 transcripts. Data underwent a series of stringent statistical analyses (see details in Materials and methods). The hierarchical clustering of the microarray data showed that pools of three independent biological replicates, namely striatal tissues from MPH- and SAL-treated rats, displayed highly similar profiles of gene expression, thus demonstrating excellent reproducibility within each treatment group. It was possible to draw the same tree clustering for the normalized data set before and after prefiltering and after statistical filter (data not shown). Stringent statistical filtering allowed the identification of 2373 striatal probesets that differed in expression between MPH- and SAL-treated rats, corresponding to $15 \%$ of the screened sequences. These genes were clustered into groups by biological function, as defined by the Gene Ontology consortium (www.geneontology.org). Interestingly, the majority of affected genes encode proteins involved in mechanisms of signal transduction, transport, transcription, and neural transmission. Among the gene families significantly affected by $\mathrm{MPH}$, those potentially involved in synaptic plasticity were highly represented (ie the following Gene Ontology families: synapse organization and biogenesis, synaptogenesis, development, morphogenesis, regulation of morphogenesis, learning and memory, and cytoskeletal organization; see Table 2 in Supplementary information).

In order to retrieve relevant information from such large data sets of genes modulated by $\mathrm{MPH}$, we selected transcripts with at least a 1.5-fold higher expression compared with SAL-treated animals (754 probesets corresponding to $4.7 \%$ of the total probesets analyzed). In order to establish a relationship between behavioral changes observed after chronic MPH treatment and changes of expression of this broad class of genes, we focused our attention on classical neurotransmitter receptors and on postsynaptic-density (PSD) proteins, two families likely to be directly or indirectly related to the plastic behavioral changes observed. Among the genes belonging to the PSD family, microarray data showed that Homer1, Shank2, and the 'MAGUK p55 subfamily member 3' (Mpp3) mRNAs were all increased in the MPH-treated striata by a 1.6-fold change (see Supplementary information, Table 2). Among the genes coding for classical neurotransmitter receptors, microarray data showed that Grik2, Htr7, adrenergic receptor $(A d r) \alpha 1 b, G A B A_{A}$ receptor $\gamma 1$ subunit $(G a b R \gamma 1)$, and $\mathrm{GABA}_{\mathrm{A}}$ receptor $\beta 3$ subunit $(G a b R \beta 3)$ transcripts were increased following $\mathrm{MPH}$ treatment with a fold change of 1.6 or more (see Supplementary information, Table 2).

$R T-P C R$ validation. To validate the microarray data on the selected genes, we performed RT-PCR analyses on single (not pooled) striatal RNAs from the SAL- and the MPHtreated animals, killed $2 \mathrm{~h}$ after the last injection. These analyses were performed on samples from two independent batches of adolescent rats: the 'first' batch, also used for microarray $(n=9)$, and the 'second' batch of independently injected animals $(n=5)$ (see Materials and methods). In both analyses, results were consistently reproduced. To evaluate the long-term molecular effects of adolescent MPH administration, we performed RT-PCR analyses on striatal tissues of nine adult rats, belonging to the 'first' batch and killed in adulthood (at pnd $>90$, ie at least 8 weeks after the end of the treatment). We found a strong correlation between the expression patterns predicted by microarrays and those determined by RT-PCR analyses: all the eight upregulated genes selected from the microarray data were validated, whereas other similar genes, not varying in the microarray experiments, did not show variation by RT-PCR analyses (see below). These results strengthen the reliability of our microarray data.

The Homer1, Shank2, and Mpp3 transcripts were upregulated in the MPH-treated adolescent striatal complex (see Figure 3, panels a, c, and e). None of these modifications persisted in the adult striatal complex (see Figure 3 panels $b, d$, and $f$ ).

The Homer 1 gene has two splice variants: $1 a$ and $1 b$. They have opposite functional effects in cultured neurons, resulting in reduced or increased density and size of dendritic spines and synapses, respectively (Sala et al, 2001, 2003). Therefore, we analyzed the expression of both splice variants separately. As shown in Figure 4, Homer $1 a$ and Homer $1 b$ transcripts were differently affected by $\mathrm{MPH}$ treatment. The former did not show short-term druginduced variation in the adolescent striatal complex, but
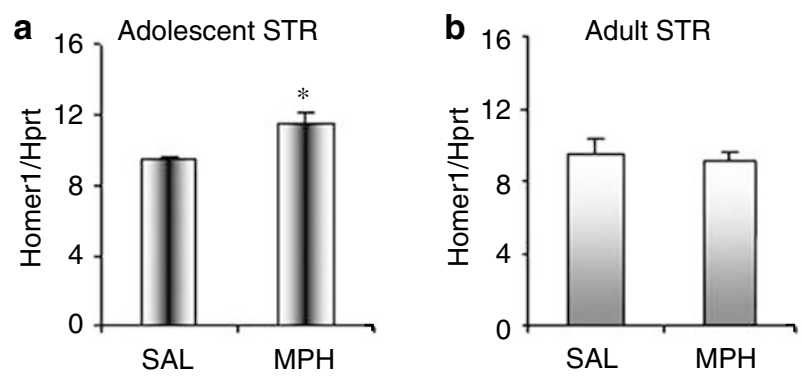

C

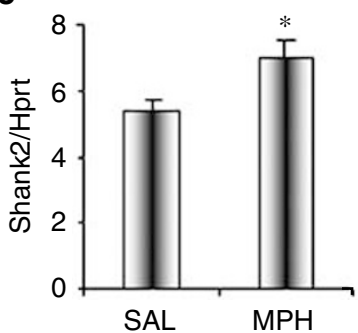

d
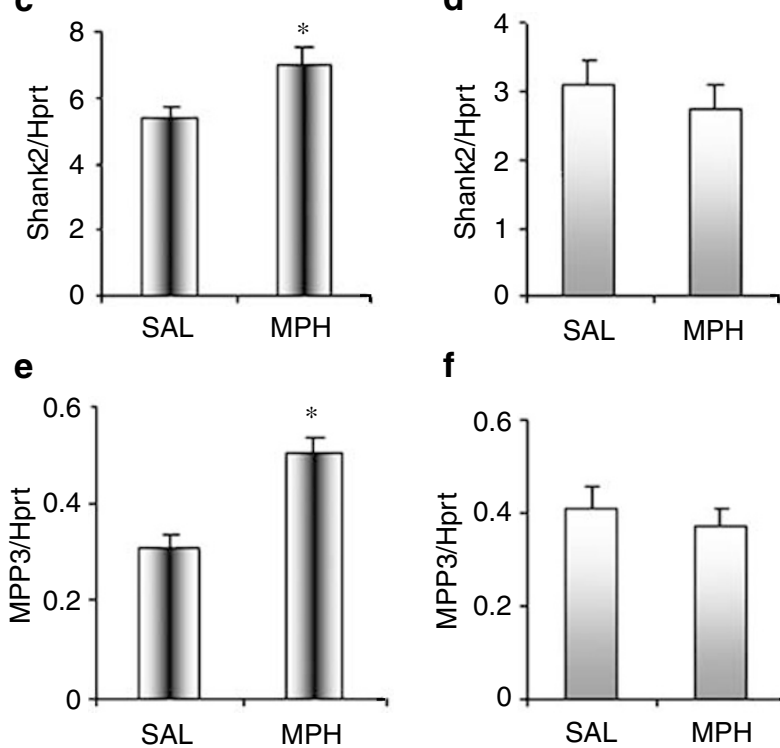

$\mathbf{f}$

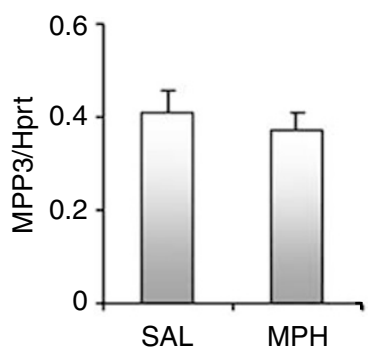

Figure 3 RT-PCR analysis of Homerl, Shank2, and MPP3 mRNAs. Samples were obtained from adolescent ( $a, c, e ; n=5)$ and adult striata (b, $d, f ; n=9$ ) from rats treated with $\mathrm{MPH}$ or SAL during adolescence (pnd $30-46$ ). The diagrams show the relative quantitation (mean \pm SEM) of the amplified products compared to that of the hypoxanthine phosphoribosyl transferase (HPRT, internal standard). * $p<0.05$ when comparing MPH with SAL treatment (Student's t-test). 

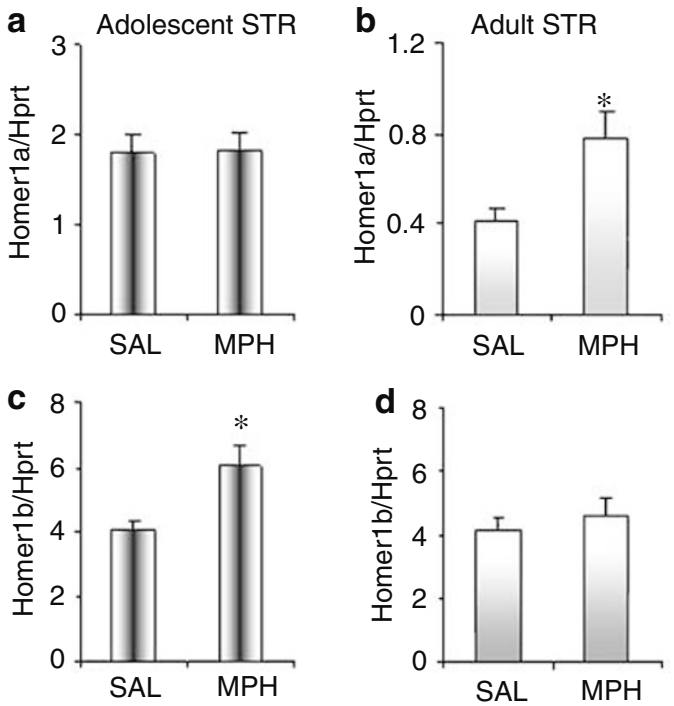

Figure 4 RT-PCR analysis of Homerl splicing variants. Samples were obtained from adolescent ( $a, c ; n=5)$ and adult striata (b, d; $n=9)$ from rats treated with MPH or SAL during adolescence (pnd 30-46). The diagrams show the relative quantitation (mean \pm SEM) of the amplified products compared to that of the hypoxanthine phosphoribosyl transferase (HPRT, internal standard). ${ }^{*} p<0.05$ when comparing $\mathrm{MPH}$ with $\mathrm{SAL}$ treatment (Student's $t$-test)

was significantly increased as enduring drug consequence in the adult (fold change 1.9); conversely, the latter was transiently increased in the adolescent (fold change 1.5), but this change did not persist in the adult striatal complex. The Homer1a upregulation did not cause a global increase of Homer1 mRNA during adulthood, probably because the expression of this splicing variant represents only a minor fraction of Homer1 transcripts. On the contrary, the upregulation of Homer $1 b$ during adolescence likely accounted for the observed global increase of Homer 1 transcripts at this age.

As for the neurotransmitter receptor genes, the Grik2, the $H t r 7$, the Adr $\alpha 1 b$, the GabR $\gamma 1$, and the GabR $\beta 3$ subunits were significantly upregulated in the MPH-treated adolescent striatal complex (Figure 5, panels a, c, e, g, and i). Interestingly, these modifications persisted in the adult striatal complex (ie 1 month after the end of the MPH treatment) only for the Grik2 and the Htr7 transcripts, but not for the other receptors (see Figure 5, panels b, d, f, h, and 1). Interestingly, both these transcripts did not vary in the adolescent or in the adult cerebellum (data not shown), thus confirming the regional specificity of the observed effects.

In addition, both microarray and RT-PCR experiments did not detect modulation either in adolescent or in adult striata for expression of other genes, belonging to the same families of those showing MPH-induced modulation, or known to be affected by other psychostimulants. This was true for Homer2, Homer3, PSD95, ionotropic glutamate receptor-A (Grial also known as GluRA), 5-hydroxytryptamine receptor 2a $(H t r 2 a)$, DA receptor 1 (Drd1), DA receptor $2(D r d 2)$, dynorphin, and the transcription factor $\triangle F o s B$ (data not shown). On the contrary, CREB transcripts were not modulated by MPH in the adolescent striatal complex (microarray and RT-PCR experiments), but were upregulated in the adult striatal complex when compared to a

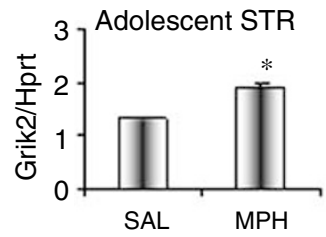

c

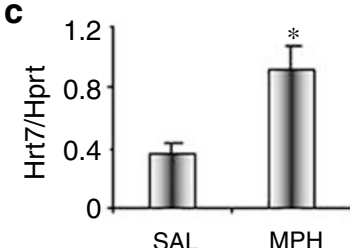

e

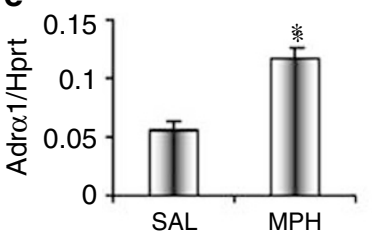

g

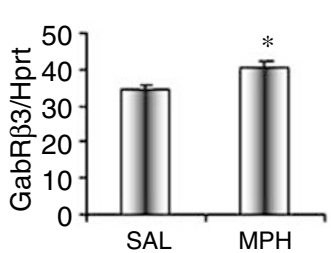

i

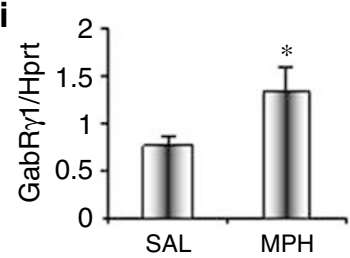

b
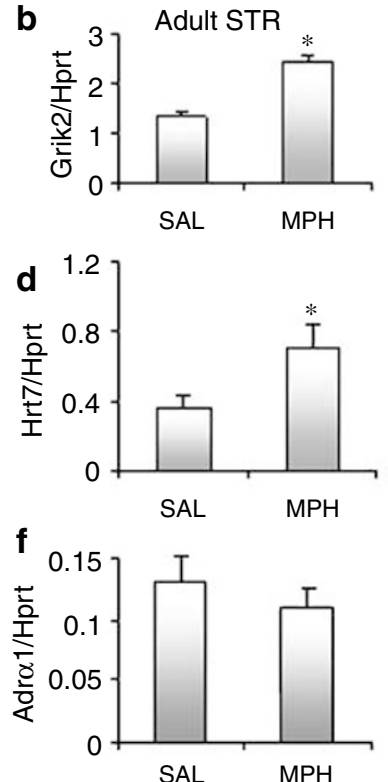

h

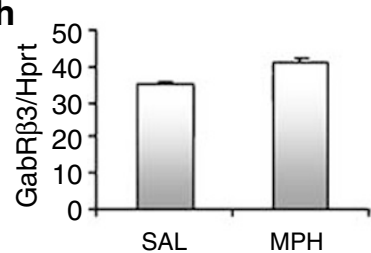

j

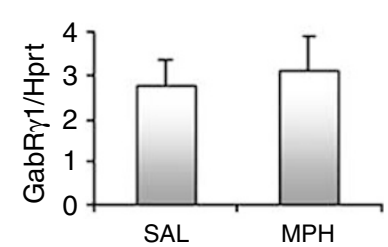

Figure 5 RT-PCR analysis of neurotransmitter receptor mRNAs. Samples were obtained from adolescent (a, c, e, g, i; $n=5$ ) and adult striata (b, d, f, h, l; $n=9$ ) from rats treated with $\mathrm{MPH}$ or $\mathrm{SAL}$ during adolescence (pnd 30-46). The diagrams show the relative quantitation (mean \pm SEM) of the amplified products (Grik2, Htr7, AdR $\alpha|b, G a b R \gamma|$, and GabR $\beta 3$ ) compared to that of the hypoxanthine phosphoribosyl transferase (HPRT, internal standard). *p $<0.05$ when comparing MPH with SAL treatment (Student's t-test).

the SAL control (see Figure 6). This result is consistent with previous data, showing that rats exposed to MPH during adolescence had increased CREB expression within the NAc shell in adulthood (Andersen et al, 2002).

Finally, none of the eight genes modulated in the arrays by subchronic MPH treatment (16 days) was altered by a single acute MPH injection during adolescence. On the contrary, the transcripts for the immediate-early genes Homer1 $a$ and $c$-fos were increased by acute MPH treatment by 1.5 - and 2.25 -fold, respectively, when compared to SALtreated rats (data not shown); these data are consistent with previous experiments (Yano and Steiner, 2004, 2005).

\section{DISCUSSION}

The aim of this study was to examine at the behavioral and molecular levels how subchronic exposure to MPH in 

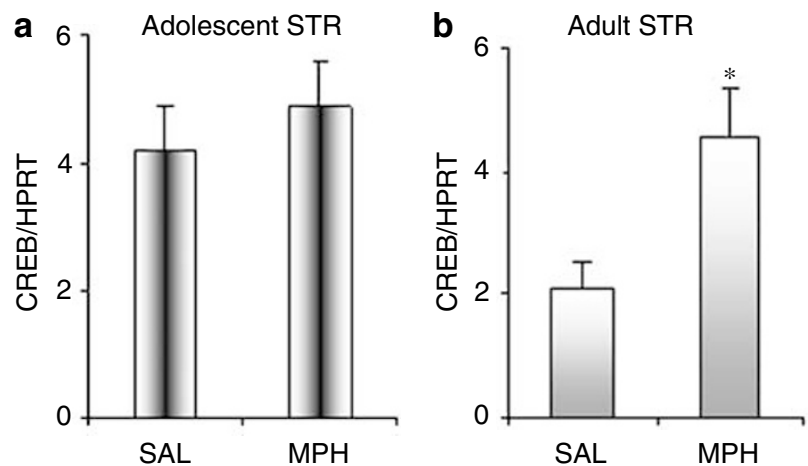

Figure 6 RT-PCR analysis of CREB mRNA. Samples were obtained from adolescent $(a ; n=5)$ and adult striata $(b ; n=9)$ from rats treated with $\mathrm{MPH}$ or SAL during adolescence (pnd 30-46). The diagram shows the relative quantitation (mean \pm SEM) of the amplified products compared to that of the HPRT (internal standard). ${ }^{*} p<0.05$ when comparing MPH with SAL treatment (Student's t-test).

adolescent rats affects neurobehavioral development, both shortly after exposure and in adulthood. To achieve this goal, we have analyzed reward-related behavioral alterations and changes in expression profiling by a genome-wide approach. Adolescent MPH pretreatment resulted in adult animals showing more flexible choice behavior. Also, COCinduced place conditioning was compromised, while COCinduced sensitization of locomotor activity was markedly increased. Expression profiling experiments showed that, among genes up-regulated in the adolescent rat striatal complex after subchronic MPH, there are members of the PSD family, potentially involved in controlling the efficiency of synaptic transmission, and members of the classical neurotransmitter receptors. Interestingly, only some of these effects were long-lasting, being still present in the adult striatal complex.

The effects observed with the genome-wide approach are a consequence of the subchronic MPH treatment (16 days). Indeed, the last injection alone cannot account for these effects, since none of the genes modulated in the arrays was affected in the acute-treatment condition. However, the latter treatment did exert a stimulatory effect on the expression of immediate early genes, such as Homer $1 a$ and $c$-fos, according to recent data (Yano and Steiner, 2004, 2005). Contrary to other reports (Brandon and Steiner, 2003), we did not observe increased expression of dynorphyn, probably due to differences in the age of animals, length, and dose of subchronic MPH administration. Consistent with the literature data, we did observe increased CREB expression only in adulthood (Andersen et al, 2002).

\section{Cocaine-Induced Place Conditioning and Sensitization}

Long-term consequences of adolescent MPH exposure led to an important dissociation between the rewarding and the arousing power of cocaine. Specifically, the incentive memory of COC-induced pleasurable effects was reduced, according to available literature (Andersen et al, 2002, but see also Brandon et al, 2001). On the other hand, druginduced locomotor sensitization was markedly potentiated. Such a finding raises some concern about the safety of MPH prescription to ADHD children. Indeed, behavioral sensitization has been implied in the pathogenesis of compulsive drug-seeking habits (Stewart and Badiani, 1993; Robinson and Berridge, 1993; Gerdeman et al, 2003). The findings of enhanced psychomotor effect and lower rewarding efficacy of $\mathrm{COC}$ are not in contrast: these two phenomena are served by distinct DA pathways and receptors (Robbins and Everitt, 1996; Wise, 2002). Strikingly, a similar picture, consisting of increased sensitization and decreased place conditioning, has been suggested as a characteristic trait of adolescence (Adriani and Laviola, 2002, 2003). We may hence suggest that adolescent MPH exposure resulted in adult individuals showing persistence of some adolescent behavioral features (Spear, 2000). The latter include a basal tone of unsatisfaction and anhedonia, due to reduced reward sensitivity (Laviola et al, 2003; Tirelli et al, 2003). The depressive-like status, found in rats after adolescent MPH exposure (Carlezon et al, 2003; Bolanos et al, 2003), seems highly consistent with this proposed interpretation.

\section{Choice Behavior}

When testing adolescent animals, in a range of ' $p$ ' values above the Indifference Point (see Materials and methods), control subjects showed increased H5 demands and a clear tendency towards disappearance of $\mathrm{H} 1$ responses, which attained a floor level. It should be noted that, with this range of ' $p$ ' values, H5 shifting was actually more convenient than $\mathrm{H} 1$ shifting. Indeed, by further increasing $\mathrm{H} 5$ responding, control animals apparently tried to compensate for the drop in large-reward delivery. Consistently, it has been shown that the proportion of 'risky' choices does increase as the scheduled availability of a feeding resource decreases (Hastjarjo et al, 1990; Kaminski and Ator, 2001). In other words, control rats showed an economically forced shift in their choice behavior. Interestingly, MPH-treated subjects did not apparently react to the stochastic-rarefaction challenge and were less dependent upon being actually food-rewarded, possibly due to direct MPH-induced hedonic activation (Marsteller et al, 2002). In conclusion, in agreement with similar previous evidence (Adriani et al, 2004), a subchronic MPH administration allowed adolescent animals to increase their ability to cope with adverse reinforcement contingencies, represented here by the unpredictable omission of large-reward delivery.

When animals were re-tested in a drug-free state in adulthood, a more severe challenge was introduced (' $p$ ' level $<20 \%$, beyond the point of 'economical indifference', see Materials and methods). Control rats maintained their preference for $\mathrm{H} 5$, and continued to choose the five-pellet reward, despite almost $80 \%$ of $\mathrm{H} 5$ nose-poking not triggering food delivery, with an average waiting time of around $100 \mathrm{~s}$. In other words, receiving a larger reward eventually and all at once continued to be preferred over receiving a certain but smaller reward, and this in spite of adverse long-term consequences on total foraging. This observation is consistent with preliminary evidence in our lab, suggesting a strong 'instinctive' preference for binge reinforcement in naive food-restricted rats (Hastjarjo et al, 1990; Kaminski and Ator, 2001). As an alternative explanation, adult control rats expressed a kind of fixed habit-based responding. Accordingly, recent findings suggest that adult individuals are less flexible than adolescents (Laviola et al, 2003). While control rats were quite insensitive to the 
schedule-related challenge, their MPH-pretreated siblings quickly displayed a marked reaction against decreased frequency of large reinforcement. An increase in H1 responding, shown by MPH-pretreated subjects, is only apparently consistent with enhanced impulsivity (Mobini et al, 2000). Indeed, the present enduring effects of adolescent MPH shall be interpreted in terms of (a) increased 'economical' efficiency, and/or (b) enhanced behavioral flexibility. While the latter is possibly due to an improvement of striatal plasticity (see below), the former feature may result from a lower impact of 'subcortical' drives (the binge-reward preference) and/or a more careful 'cortical' evaluation of the long-term payoff (McClure $e t$ al, 2004; Ridderinkhof et al, 2004). MPH-exposed rats may perceive the 'global' effects of accumulating omitted rewards (the 'losses') across time, rather than being merely driven by the 'unitary' size of the eventual outcome (the binge but rare 'wins'). Alternatively, MPH-pretreated rats may be less dependent on established behavioral habits compared to the SAL-pretreated siblings. Interestingly, enhanced flexibility may represent another 'adolescent-like' feature in adult rats pre-exposed to MPH.

In summary, the presence or absence of a preference shift, shown by rats when re-tested in adulthood, is discussed here in terms of flexibility and economical efficiency of the choice behavior. These functional parameters may be influenced by a balance between (a) subcortical bingereward preference and/or established behavioral habits, which would both promote response perseverance, and (b) cortical evaluation of actual outcomes (ie impact of omitted rewards on long-term pay off), which would promote a flexible response shift. Integration of such opposite drives might occur within a crosstalk between the striatal DA pathways and the serotonergic systems of the prefrontal cortex (Ragozzino, 2003; Yin et al, 2004; McClure et al, 2004; Ridderinkhof et al, 2004).

\section{Gene-Expression Changes}

Enduring behavioral changes caused by repeated exposure to psychoactive drugs, such as sensitization, are likely to involve changes in gene expression within the mesocorticolimbic DA pathway. The striatal complex was chosen for microarray experiments as a representative target area of this reward-related circuit, since molecular changes induced by acute MPH treatment have been observed in most sectors of the rat caudate putamen (Yano and Steiner, 2004). By genome-wide expression profiling, followed by RT-PCR validation, eight genes, belonging to two families, show significant changes in their striatal expression at the end of the MPH treatment: three PSD genes (Homer1, Shank2, and MPP3), and five neurotransmitter receptors (Grik2, Gabr 1 , Gabrß3, Htr7, and Adr $\alpha 1 b$ ).

The PSD family forms a network of interacting proteins anchoring and linking neurotransmitter receptors and other postsynaptic membrane proteins to cytoskeletal elements and signaling pathways (Scannevin and Huganir, 2000). Many of these genes have been involved in processes related to long-term synaptic plasticity in the striatum, and psychostimulants are known to exert a pronounced influence on these processes (Nestler, 2001; Gerdeman et al, 2003). Homer and Shank proteins are core compo- nents of the PSD family, interacting directly with each other and being associated with the NMDA receptor and with type I-metabotropic GluRs (Scannevin and Huganir, 2000). Interestingly, members of the Homer subfamily are modulated by acute and chronic cocaine administration, and regulate sensitivity to this drug (Szumlinski et al, 2004). The MPP3 gene belongs to the MAGUK subfamily of synaptic membrane-associated proteins, involved in functions that modulate NMDA receptors, including synaptic targeting, clustering, and coupling to second-messenger signaling systems (Scannevin and Huganir, 2000).

$\mathrm{MPH}$-induced changes in PSD genes could act through regulation of synaptic efficacy and (glutamate) receptor function (targeting, clustering, coupling to second messengers, etc), and/or through structural alterations, like those observed in dendrite morphology following repeated administration of psychostimulants (Robinson and Kolb, 2004). Indeed, Homer and Shank2 cooperate to induce structural and functional organization of dendritic spines and synapses (Sala et al, 2001, 2003). Thus, our data showing coordinated upregulation of transcripts for both Homer1 and Shank2 strongly suggest that remodeling of dendritic spines could occur during adolescence following subchronic MPH treatment. The splice variant Homer $1 b$ (which cooperates with Shank to induce morphological growth and maturation; Sala et al, 2001) was not modulated in adulthood. Conversely, the splice variant Homerla (which reduces the density and size of dendritic spines by inhibition of Shank targeting to the synapses; Sala et al, 2003) was upregulated only in the adult striatal complex. We hypothesize that these two specific MPH effects on Homer1 splice variants might underlie opposite consequences: structural growth and functional improvement of striatal circuits during MPH treatment in adolescence, and conversely a reduction of synaptic size and efficacy as a long-term consequence of adolescent MPH treatment in the adult brain. Future morphological and electrophysiological analyses are needed to test this hypothesis.

Data show that chronic MPH administration, which is thought to increase extracellular DA in the ventral striatum by blocking the DA transporter (Swanson and Volkow, 2002), alters the expression of ionotropic receptor subunits (Grik2, GabR $\gamma 1$, and GabR/3) and G-coupled receptors ( $H$ tr7 and Adra $1 b$ ), thus involving various classical neurotransmitters (glutamate, GABA, serotonin, and noradrenaline). However, only Grik2 and Htr7 transcripts were still upregulated in adulthood. Hence, most of the neurotransmission of the basal ganglia circuits is affected at the end of the treatment and only some of these alterations are long-lasting. In the striatum, a dynamic communication is established between midbrain DA afferents and cortical glutamatergic terminals, which converge on GABAergic spiny projection neurons (Smith and Bolam, 1990). Glutamate and GABA receptors are pivotal in the induction, expression, and/or modulation of synaptic plasticity (Collingridge et al, 2004). Thus, alterations of glutamatergic and/or GABAergic receptor subunits following chronic MPH treatment were expected. Moreover, psychostimulants also affect the excitability of DA neurons via the noradrenergic and serotonergic systems (Auclair et al, 2004; Paladini et al, 2004). Recent experiments implicate Adralb in DA release, locomotion, and behavioral 
sensitization induced by psychostimulants (Drouin et al, 2002; Battaglia et al, 2003). The increased striatal expression of $A d r \alpha 1 b$ following $\mathrm{MPH}$ administration supports its critical role in the behavioral and molecular effects of psychostimulants.

Recent data demonstrate that Grik2 subunits are important modulators of heterosynaptic facilitation (Contractor et al, 2003, 2001), and that significant Grik2 alterations are linked to reward dysfunctions (Tang et al, 2004, 2003).

It is well established that the serotonergic pathways play a role in timing behavior (Wogar et al, 1993; Morrissey et al, 1994), and either reduced or increased serotonin function may cause tendencies towards perseverative behavior (Dalley et al, 2002; Puumala and Sirvio, 1998; Harrison et al, 1997; Wolff and Leander 2002). In addition, genetic knockout and pharmacological blockade studies of the Htr7 receptor suggest its potential involvement in depression (Guscott et al, 2005). Thus, the persistent modulation of Htr7 gene expression after chronic MPH treatment is particularly intriguing. A key role of $\mathrm{Htr} 7$ receptors in behavioral flexibility and/or reward-evaluation processes is proposed. This hypothesis deserves future neuro-pharmacological analyses.

Further experiments will establish a causal functional link between enduring changes in the expression levels of Grik2 or Htr7 and the observed behavioral phenotype.

To our knowledge, this is the first study that combines a behavioral analysis and a genome-wide approach, aimed to investigate the molecular bases of neurobehavioral changes due to chronic MPH exposure during adolescence. In summary, our observations indicate that exposure to MPH during adolescence is able to modulate striatal gene expression of members of the PSD and neurotransmitterreceptor families. We suggest that these alterations underlie the fundamental plastic structural and functional plastic changes in striatal circuits. These changes may in turn account for alterations in processing of incentive information and in flexibility/habit balance.

\section{ACKNOWLEDGEMENTS}

This research was supported by the Project 'Pathogenesis and recovery in animal and in-vitro models of Alzheimer disease', Italian Ministry of Health; by MURST/MIUR Cofin. 2003 (No. 2003059030_002); by FIRB Neuroscienze (No. RBNE01WY7P); and by the EU FP5 grant QLRT-200101000-GDNF. We thank Dr S Crispi and Dr P De Luca for their help in the microarray experiments, M Sbragi for operant-chamber computer software, and $M$ Terracciano and $\mathrm{L}$ Leone for their technical assistance. We disclose any involvement (financial or otherwise) that might potentially bias our work.

\section{REFERENCES}

Adriani W, Laviola G (2002). Spontaneous novelty seeking and amphetamine-induced conditioning and sensitization in adult mice: evidence of dissociation as a function of age at weaning. Neuropsychopharmacology 27: 225-236.

Adriani W, Laviola G (2003). Elevated levels of impulsivity and reduced place conditioning with $\mathrm{D}$-amphetamine: two behavioral features of adolescence in mice. Behav Neurosci 117: 695-703.
Adriani W, Rea M, Baviera M, Invernizzi W, Carli M, Ghirardi O et al (2004). Acetyl-L-carnitine reduces impulsive behaviour in adolescent rats. Psychopharmacology 176: 296-304.

Andersen SL, Arvanitogiannis A, Pliakas AM, LeBlanc C, Carlezon Jr WA (2002). Altered responsiveness to cocaine in rats exposed to methylphenidate during development. Nat Neurosci 5: 13-14.

Auclair A, Drouin C, Cotecchia S, Glowinski J, Tassin JP (2004). 5-HT2A and alphalb-adrenergic receptors entirely mediate dopamine release, locomotor response and behavioural sensitization to opiates and psychostimulants. Eur J Neurosci 20: 30733084.

Battaglia G, Fornai F, Busceti CL, Lembo G, Nicoletti F, De Blasi A (2003). Alpha-1B adrenergic receptor knockout mice are protected against methamphetamine toxicity. J Neurochem 86: 413-421.

Bolanos CA, Barrot M, Berton O, Wallace-Black D, Nestler EJ (2003). Methylphenidate treatment during pre- and periadolescence alters behavioral responses to emotional stimuli at adulthood. Biol Psychiatry 54: 1317-1329.

Brandon CL, Marinelli M, Baker LK, White FJ (2001). Enhanced reactivity and vulnerability to cocaine following methylphenidate treatment in adolescent rats. Neuropsychopharmacology 25: 651-661.

Brandon CL, Steiner H (2003). Repeated methylphenidate treatment in adolescent rats alters gene regulation in the striatum. Eur J Neurosci 18: 1584-1592.

Carlezon Jr WA, Konradi C (2004). Understanding the neurobiological consequences of early exposure to psychotropic drugs: linking behavior with molecules. Neuropharmacology 47: 47-60.

Carlezon Jr WA, Mague SD, Andersen SL (2003). Enduring behavioral effects of early exposure to methylphenidate in rats. Biol Psychiatry 54: 1330-1337.

Collingridge GL, Isaac JT, Wang YT (2004). Receptor trafficking and synaptic plasticity. Nat Rev Neurosci 5: 952-962.

Contractor A, Sailer AW, Darstein M, Maron C, Xu J, Swanson GT et al (2003). Loss of kainate receptor-mediated heterosynaptic facilitation of mossy-fiber synapses in KA2-I- mice. J Neurosci 23: 422-429.

Contractor A, Swanson G, Heinemann SF (2001). Kainate receptors are involved in short- and long-term plasticity at mossy fiber synapses in the hippocampus. Neuron 29: 209-216.

Dalley JW, Theobald DE, Eagle DM, Passetti F, Robbins TW (2002). Deficits in impulse control associated with tonically-elevated serotonergic function in rat prefrontal cortex. Neuropsychopharmacology 26: 716-728.

Drouin C, Darracq L, Trovero F, Blanc G, Glowinski J, Cotecchia S et al (2002). Alphalb-adrenergic receptors control locomotor and rewarding effects of psychostimulants and opiates. J Neurosci 22: 2873-2884.

Evenden JL (1999). Varieties of impulsivity. Psychopharmacology 146: 348-361.

Gerdeman GL, Partridge JG, Lupica CR, Lovinger DM (2003). It could be habit forming: drugs of abuse and striatal synaptic plasticity. Trends Neurosci 26: 184-192.

Glimcher PW, Rustichini A (2004). Neuroeconomics: the consilience of brain and decision. Science 306: 447-452.

Guscott M, Bristow LJ, Hadingham K, Rosahl TW, Beer MS, Stanton JA et al (2005). Genetic knockout and pharmacological blockade studies of the 5-HT7 receptor suggest therapeutic potential in depression. Neuropharmacology 48: 492-502.

Harrison AA, Everitt BJ, Robbins TW (1997). Central 5-HT depletion enhances impulsive responding without affecting the accuracy of attentional performance: interactions with dopaminergic mechanisms. Psychopharmacology 133: 329-342.

Hastjarjo T, Silberberg A, Hursh SR (1990). Risky choice as a function of amount and variance in food supply. J Exp Anal Behav 53: 155-161. 
Kaminski BJ, Ator NA (2001). Behavioral and pharmacological variables affecting risky choice in rats. J Exp Anal Behav 75: 275297.

Laviola G, Macri S, Morley-Fletcher S, Adriani W (2003). Risktaking behavior in adolescent mice: psychobiological determinants and early epigenetic influence. Neurosci Biobehav Rev 27: 19-31.

Laviola G, Wood RD, Kuhn C, Francis R, Spear LP (1995). Cocaine sensitization in periadolescent and adult rats. J Pharmacol Exp Ther 275: 345-357.

Marsteller DA, Gerasimov MR, Schiffer WK, Geiger JM, Barnett CR, Borg JS et al (2002). Acute handling stress modulates methylphenidate-induced catecholamine overflow in the medial prefrontal cortex. Neuropsychopharmacology 27: 163-170.

McClure SM, Laibson DI, Loewenstein H, Cohen JD (2004). Separate neural systems value immediate and delayed monetary rewards. Science 306: 503-507.

Mobini S, Chiang TJ, Al-Ruwaitea AS, Ho MY, Bradshaw CM, Szabadi E (2000). Effect of central 5-hydroxytryptamine depletion on inter-temporal choice: a quantitative analysis. Psychopharmacology 149: 313-318.

Montague PR, Berns GS (2002). Neural economics and the biological substrates of valuation. Neuron 36: 265-284.

Morrissey G, Ho MY, Wogar MA, Bradshaw CM, Szabadi E (1994). Effect of lesions of the ascending 5-hydroxytryptaminergic pathways on timing behaviour investigated with the fixedinterval peak procedure. Psychopharmacology 114: 463-468.

Nestler EJ (2001). Molecular basis of long-term plasticity underlying addiction. Nat Rev Neurosci 2: 119-128.

Nestler EJ (2004). Molecular mechanisms of drug addiction. Neuropharmacology 47: 24-32.

Paladini CA, Mitchell JM, Williams JT, Mark GP (2004). Cocaine self-administration selectively decreases noradrenergic regulation of metabotropic glutamate receptor-mediated inhibition in dopamine neurons. J Neurosci 24: 5209-5215.

Puumala T, Sirvio J (1998). Changes in activity of dopamine and serotonin systems in the frontal cortex underlie poor choice accuracy and impulsivity of rats in an attention task. Neuroscience 83: 489-499.

Ragozzino ME (2003). Acetylcholine actions in the dorsomedial striatum support the flexible shifting of response patterns. Neurobiol Learn Mem 80: 257-267.

Ridderinkhof KR, van der Wildenberg WPM, Segalowitz SJ, Carter CS (2004). Neurocognitive mechanisms of cognitive control: the role of prefrontal cortex in action selection, response inhibition, performance monitoring and reward-based learning. Brain Cognition 56: 129-140.

Robbins TW, Everitt BJ (1996). Neurobehavioural mechanisms of reward and motivation. Curr Opin Neurobiol 6: 228-236.

Robinson TE, Berridge KC (1993). The neural basis of drug craving: an incentive-sensitization theory of addiction. Brain Res Brain Res Rev 18: 247-291.

Robinson TE, Kolb B (2004). Structural plasticity associated with exposure to drugs of abuse. Neuropharmacology 47: 33-46.

Sagvolden T, Sergeant JA (1998). Attention deficit/hyperactivity disorder - from brain dysfunction to behavior. Behav Brain Res 94: $1-10$.

Sala C, Futai K, Yamamoto K, Worley PF, Hayashi Y, Sheng M (2003). Inhibition of dendritic spine morphogenesis and synaptic transmission by activity-inducible protein Homerla. $J$ Neurosci 23: 6327-6337.
Sala C, Piech V, Wilson NR, Passafaro M, Liu G, Sheng M (2001). Regulation of dendritic spine morphology and synaptic function by Shank and Homer. Neuron 31: 115-130.

Scannevin RH, Huganir RL (2000). Postsynaptic organization and regulation of excitatory synapses. Nat Rev Neurosci 1: 133-141.

Schultz W (2004). Neural coding of basic reward terms of animal learning theory, game theory, microeconomics and behavioural ecology. Curr Opin Neurobiol 14: 139-147.

Smith AD, Bolam JP (1990). The neural network of basal ganglia as revealed by the study of synaptic connections of identified neurons. Trends Neurosci 13: 259-265.

Sonuga-Barke EJ (2003). The dual pathway model of AD/HD: an elaboration of neuro-developmental characteristics. Neurosci Biobehav Rev 27: 593-604.

Spear LP (2000). The adolescent brain and age-related behavioral manifestations. Neurosci Biobehav Rev 24: 417-463.

Stewart J, Badiani A (1993). Tolerance and sensitization to the behavioral effects of drugs. Behav Pharmacol 4: 289-312.

Swanson J, Castellanos FX, Murias M (1998). Cognitive neuroscience of attention deficit hyperactivity disorder and hyperkinetic disorder. Curr Opin Neurobiol 8: 263-271.

Swanson JM, Volkow ND (2002). Pharmacokinetic and pharmacodynamic properties of stimulants: implications for the design of new treatments for ADHD. Behav Brain Res 130: 73-78.

Szumlinski KK, Dehoff MH, Kang SH, Frys KA, Lominac KD, Klugmann $\mathrm{M}$ et al (2004). Homer proteins regulate sensitivity to cocaine. Neuron 43: 401-413.

Tang W, Wesley M, Freeman WM, Liang B, Hemby SE (2004). Alterations in ionotropic glutamate receptor subunits during binge cocaine self-administration and withdrawal in rats. $J$ Neurochem 89: 1021-1033.

Tang WX, Fasulo WH, Mash DC, Hemby SE (2003). Molecular profiling of midbrain dopamine regions in cocaine overdose victims. I Neurochem 85: 911-924.

Tirelli E, Laviola G, Adriani W (2003). Ontogenesis of behavioral sensitization and conditioned place preference induced by psychostimulants in laboratory rodents. Neurosci Biobehav Rev 27: $163-178$.

Wise RA (2002). Brain reward circuitry: insights from unsensed incentives. Neuron 36: 229-240.

Wogar MA, Bradshaw CM, Szabadi E (1993). Effect of lesions of the ascending 5-hydroxytryptaminergic pathways on choice between delayed reinforcers. Psychopharmacology 111: 239-243.

Wolff MC, Leander JD (2002). Selective serotonin reuptake inhibitors decrease impulsive behavior as measured by an adjusting delay procedure in the pigeon. Neuropsychopharmacology 27: 421-429.

Yano M, Steiner H (2004). Topography of methylphenidate (Ritalin)-induced gene regulation in the striatum: differential effects on c-Fos, substance P and opioid peptides. Neuropsychopharmacology 22: 1-15.

Yano M, Steiner H (2005). Methylphenidate (Ritalin) induces Homer $1 \mathrm{a}$ and zif 268 expression in specific corticostriatal circuits. Neuroscience 132: 855-865.

Yin HH, Knowlton BJ, Balleine BW (2004). Lesions of dorsolateral striatum preserve outcome expectancy but disrupt habit formation in instrumental learning. Eur J Neurosci 19: 181-189.

Zorrilla EP (1997). Multiparous species present problems (and possibilities) to developmentalists. Dev Psychobiol 30: 141-150.

Supplementary Information is available on the Neuropsychopharmacology website (http://www.nature.com/npp). 